\title{
Indwelling pleural catheters for recurrent pleural effusions: A useful clinical tool with serious implications
}

\author{
Samuel Copeland MD, Audra Schwalk MD, Sakolwan Suchartlikitwong MD, \\ Shengping Yang PhD, Gilbert Berdine MD
}

\begin{abstract}
Background: Indwelling pleural catheters (IPC) have been used increasingly in patients with recurrent pleural effusions. However, data about mortality after IPC use are limited.

Objectives: We sought to determine the natural history following IPC placement in Lubbock, Texas, in terms of life expectancy and pleurodesis rates in patients with both malignant and benign effusions.

Methods: A retrospective review of patients who had IPC insertion from March 2014 through December 2016 at University Medical Center in Lubbock, Texas, was performed. Patients 18 years and older who had IPC placement for recurrent pleural effusions were included. The duration of IPC placement, the type of pleural effusion, the volume of fluid, pleurodesis, complications, and mortality after IPC placement were retrieved from electronic medical records.

Results: There were 45 patients included in the study; 20 patients (44\%) were male, and 25 patients (56\%) were female. The mean age was 63.5 years old. There were 33 patients with malignant pleural effusion and 12 patients with benign pleural effusion. No patients with malignant effusion were known to be alive at the time of mortality calculation, whereas two patients with benign effusion were known to be alive. Median survival was 468 days in the benign effusion group and 115 days in the malignant effusion group. The 30-day mortality was not significantly different between the two groups (malignant $34.5 \%$ vs. benign $25.0 \%$ ). However, 1-year mortality was significantly higher in the malignant effusion group (89.7\%) than in the benign effusion group (41.7\%) $(p<0.005)$.

Conclusion: The use of indwelling pleural catheters in Lubbock, Texas, has comparable results to published studies. These catheters should be considered as a bridge to a long-term treatment rather than a definitive therapy.
\end{abstract}

Keywords: Recurrent pleural effusion, indwelling pleural catheter, mortality, survival, pleurodesis

\section{INTRODUCTION}

Pleural effusion is a common clinical problem, with an incidence of roughly 3,000 people per 1 million

Corresponding author: Gilbert G. Berdine

Contact Information: Gilbert.berdine@ttuhsc.edu

DOI: $10.12746 /$ swrccc.v6i25.477 population each year. ${ }^{1}$ Pleural effusions have multiple causes; the vast majority of cases are caused by congestive heart failure, pneumonia, or malignancy. ${ }^{1}$ Symptoms generally consist of dyspnea, chest pain, or cough which can be refractory, severe, and debilitating. Therapeutic interventions are aimed at treatment of the underlying cause, control of symptoms, and prevention of recurrence. Thoracentesis may relieve acute symptoms and may assist with the diagnosis as to the cause of the effusion. The underlying 
disease may not be treatable, or the time frame for resolution may be slow; both situations may result in recurrent pleural effusions which can be difficult to treat. ${ }^{2}$ For recurrence, the focus has been on preventing fluid re-accumulation with pleurodesis. Historically this was achieved with talc instilled into the pleural space; however, this procedure carries a risk for respiratory complications and is associated with longer hospital stays. ${ }^{3}$ The use of an indwelling pleural catheter (IPC) is increasingly being used as an alternative treatment approach. This procedure can be performed in the outpatient setting, and it offers as needed drainage for symptoms, controlled by the patient from home. Indwelling pleural catheters have been established as an effective means to reduce symptoms of pleural effusions associated with malignant pleural effusions. ${ }^{4}$ Recently, the use of IPC for recurrent benign conditions, such as congestive heart failure and hepatic hydrothorax, has increased in number at our institution. The analysis of IPC associated with these benign conditions has been limited to retrospective reviews and case series. Recently a systematic review and meta-analysis analyzed outcomes in 325 patients with benign effusions. ${ }^{5}$ However, as the authors point out, the trials that made up the meta-analysis represent low quality evidence, but they are currently the best available data on this topic. This manuscript examines our experience with IPC for the management of both malignant and benign recurrent pleural effusions at Texas Tech University Health Sciences Center (TTUHSC) from March 2014 to December 2016. Specifically, we hoped to better define the natural history following IPC placement with regards to life expectancy and pleurodesis rates.

\section{Methods}

The medical records of all patients older than 18 years who had an IPC placed by the Division of Pulmonary and Critical Care at TTUHSC, Lubbock, Texas, between March 2014 and December 2016 were reviewed retrospectively. The study cohort included patients at University Medical Center and TTUHSC identified from billing data using a specific charge code for the insertion of an IPC. Patients were followed until death. Ancestry.com was used to assess the Social Security Administration death master file of patients lost to follow-up, as described previously by Peikert et al. ${ }^{6}$ Patients were included if they were older than 18 years at the time of IPC placement, and they had a diagnosis of recurrent pleural effusion. We excluded prisoners from this study. Malignant effusions were defined by the presence of malignant cells in the pleural fluid or by recurrent pleural effusions in a patient with a known malignant diagnosis. Benign effusions were defined by refractory effusions in which the person did not have a known or suspected underlying malignancy. For patients who were lost to follow up we used the electronic medical record to determine the date that the patient was last known to be alive. The study was approved by the Institutional Review Board at TTUHSC (IRB \# L17-113).

Clinical data extracted from the medical record included the date of IPC placement, the date of death, the date of last follow-up if the patient were still alive, the date of pleurodesis if this event occurred, complications, additional pleural procedures, primary diagnosis, suspected cause of pleural effusion, initial pleural fluid characteristics (WBC count and differential, RBC count, pleural fluid LDH and protein, serum LDH and protein), and the size of the pleural effusion defined as volume occupying of the chest in thirds. A single expert reader assigned a pleural effusion volume at the time of the original IPC placement as $1 / 3,2 / 3$, or $3 / 3$ of the volume of the hemithorax. Spontaneous pleurodesis was defined as drainage less than $50 \mathrm{ml}$ on consecutive drainages (at least 24 hours and usually 48 hours) and a chest radiograph demonstrating no significant accumulation of pleural fluid.

SAS software (Version 9.4) was used for statistical analyses. Student's t-test or the Mann Whitney U test was used to compare numerical variables between groups as appropriate. Chi square and Fisher's exact test were used to compare categorical variables. $P$ values less than 0.05 were considered to be statistically significant. The Kaplan-Meier method was used to estimate survival probability, and the log rank test was used to make the comparison between malignant and benign pleural effusion patients. 


\section{RESULTS}

Table 1 lists the underlying diagnoses for the benign and malignant effusions in each patient. Table 2 shows the data for the study population. Data are presented either as mean \pm SD or median $\left(25^{\text {th }}\right.$ percentile, $75^{\text {th }}$ percentile). Forty-five patients were included; 20 patients (44\%) were male, and 25 patients (56\%) were female. The mean age was $63.5 \pm 15$ years old across the study population. Malignant effusions were present in 33 patients; 12 patients had benign effusions. Differences in sex and age between the malignant effusion group and benign effusion group were not significant. The pleural fluid LDH and WBC measurements were higher in the malignant effusion patients. There were no other statistically significant differences in pleural fluid lab values between the malignant effusions and benign effusions.

While the study period ended December 2016, mortality calculations were performed based on patient status as of March 2018. All IPCs were

Table 1. Etiology of pleural effusions

\begin{tabular}{|l|c|}
\hline Type of effusion & Total \\
\hline & $\mathbf{4 5}$ \\
\hline Malignant & $\mathbf{3 3}$ \\
\hline Small cell lung cancer & 4 \\
\hline NSCLC & 11 \\
\hline Breast cancer & 8 \\
\hline Melanoma & 1 \\
\hline Lymphoma & 2 \\
\hline Colon cancer & 2 \\
\hline Ewing's sarcoma & 1 \\
\hline Renal cell carcinoma & 2 \\
\hline Uterine cancer & 1 \\
\hline Pancreatic cancer & 1 \\
\hline Benign & $\mathbf{1 1}$ \\
\hline Liver cirrhosis & 3 \\
\hline Congestive heart failure & 7 \\
\hline Systemic lupus erythematosus & 1 \\
\hline Unknown & $\mathbf{1}$ \\
\hline
\end{tabular}

removed, or patients were lost to follow up before mortality calculations were performed. For the malignant effusion group, 27 patients had died. No patients were known to be alive at the time the mortality statistics were calculated (March 2018). Six patients were lost to follow up. For the benign effusion group, seven patients were known to have died, two patients were known to be alive, and five patients were lost to follow up as of the date when mortality statistics were calculated (March 2018).

\section{Pleurodesis Rates}

For catheter removal rates, the average IPC length was $74 \pm 79$ days. The median IPC duration was 41 days. Six patients with IPC were lost to follow up before the IPC was removed or the patient expired. All patients lost to follow up before IPC removal were from the malignant effusion group. The rates of spontaneous pleurodesis resulting in catheter removal were $63.6 \%$ in patients with benign effusions and $38.5 \%$ in patients with malignant effusions ( $p$-value 0.279 ). The volume of pleural effusion had a statistically significant effect on the spontaneous pleurodesis rate $(p$-value 0.01 ) (Figure 1). Pleural effusions that occupied $1 / 3$, $2 / 3$, or $3 / 3$ of hemithorax volume had $46.2 \%, 64.7 \%$, and $0 \%$ spontaneous pleurodesis rates, respectively. Only one patient had talc instilled into the pleural space. Catheter duration in the benign effusion group was significantly longer than in the malignant effusion group with an average of 393.8 days and 132.2 days, respectively ( $p$-value 0.004$)$.

\section{MoRTALITY RATES}

Figure 2 shows the survival for the patients with malignant and benign effusions. Median survival was 468 days in the benign effusion group and 115 days in the malignant effusion group. The survival was significantly worse for the malignant effusion group $(p<$ 0.001 ). Mortality at 30 days was $25.0 \%$ for the benign effusion group and $34.5 \%$ for the malignant effusion group ( $P>0.05$ ). Mortality at 1 year was $41.7 \%$ for the benign effusion group and $89.7 \%$ for the malignant effusion group; this difference was significant ( $p$ $<0.005)$. 
Table 2. Patient demographics, pleural fluid characteristics, and outcomes

\begin{tabular}{|l|l|l|l|l|}
\hline & Total & Benign & Malignant & P value \\
\hline \# patients & 45 & 12 & 33 & \\
\hline Gender & & & & \\
\hline Female & 25 & 6 & 19 & 0.910 \\
\hline Male & 20 & 6 & 14 & \\
\hline Age (years) & $63.5 \pm 15.4$ & $68.7 \pm 12.0$ & $61.6 \pm 16.2$ & 0.242 \\
\hline Pleural Fluid Glucose (mg/dL) & $106.1 \pm 53.1$ & $129.6 \pm 65.3$ & $95.4 \pm 44.0$ & 0.234 \\
\hline Pleural Fluid LDH (IU/L) & $\begin{array}{l}247.0 \\
(128.5,522.5)\end{array}$ & $\begin{array}{l}138.0 \\
(103.5,208.0)\end{array}$ & $\begin{array}{l}(148.0 \\
631.5)\end{array}$ & $\mathbf{0 . 0 2 5}$ \\
\hline Pleural Fluid Total Protein (g/dL) & $3.3 \pm 1.0$ & $2.9 \pm 1.3$ & $3.5 \pm 0.9$ & 0.097 \\
\hline Pleural Fluid RBC (/mm $\left.{ }^{\mathbf{3}}\right)$ & $\begin{array}{l}13816.0 \\
(1191.0,45428.5)\end{array}$ & $\begin{array}{l}11766.0 \\
(527.3,39156.8)\end{array}$ & $\begin{array}{l}13926.0 \\
(4066.0,45428.5)\end{array}$ & 0.384 \\
\hline Pleural Fluid WBC (/mm $\left.\mathbf{m}^{\mathbf{3}}\right)$ & $\begin{array}{l}512.0 \\
(224.5,910.5)\end{array}$ & $\begin{array}{l}194.0 \\
(82.8,733.8)\end{array}$ & $\begin{array}{l}589.0 \\
(301.0,1122.0)\end{array}$ & $\mathbf{0 . 0 2 5}$ \\
\hline Median survival (days) & 141 & 468 & 115 & $\mathbf{0 . 0 0 1}$ \\
\hline Mortality (30 day) & $32.1 \%$ & $25.0 \%$ & $34.5 \%$ & 0.458 \\
\hline Mortality (1 year) & $74.3 \%$ & $41.7 \%$ & $89.7 \%$ & $\mathbf{0 . 0 0 5}$ \\
\hline
\end{tabular}

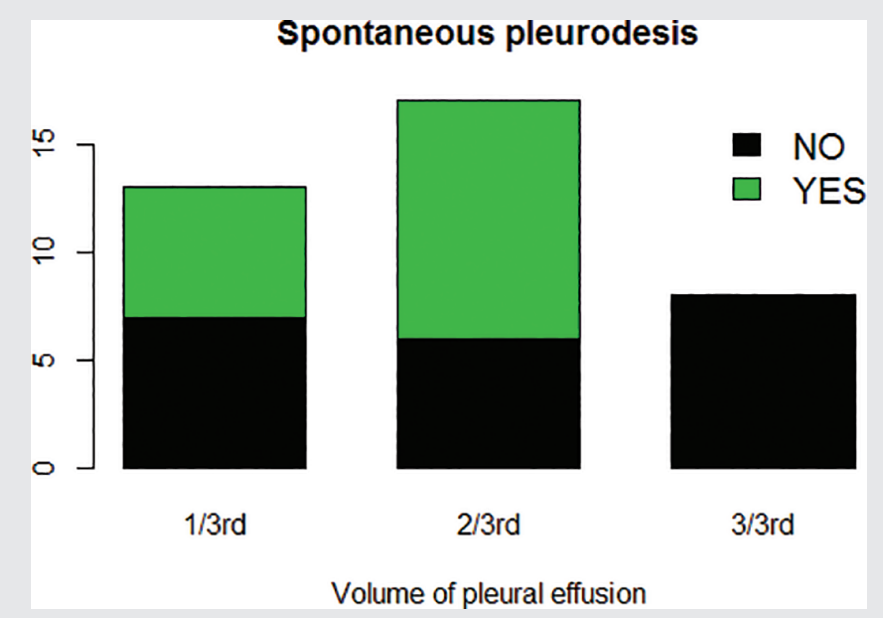

Figure 1. Spontaneous pleurodesis rates based on fluid volume.

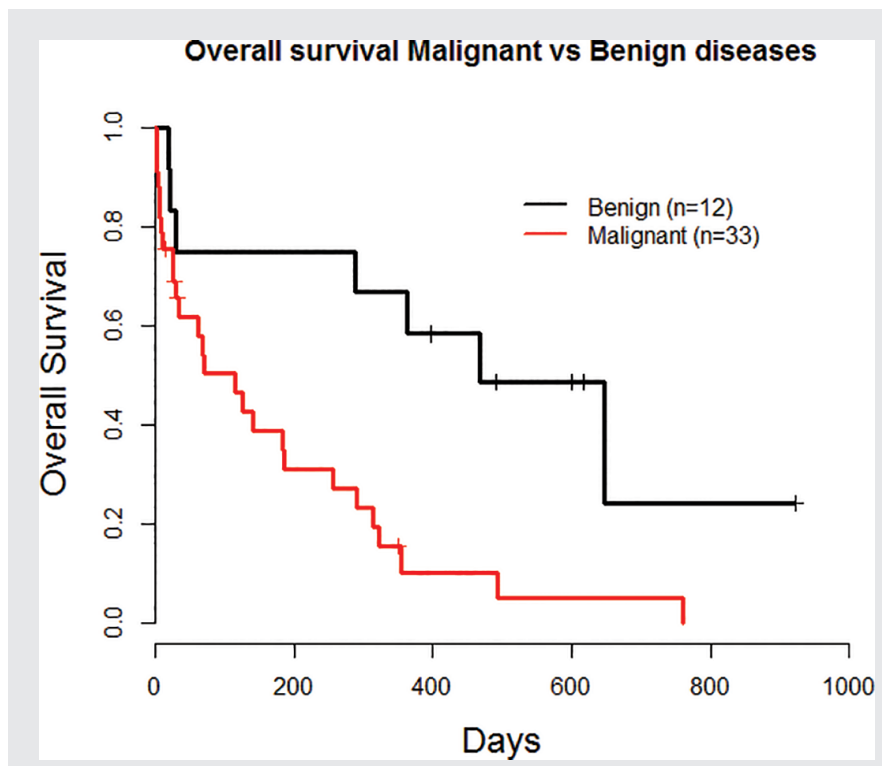

Figure 2. Mortality rates. 


\section{Discussion}

The most obvious result from our study is the high mortality rate in the malignant effusion group. The combined group 141 day median survival was consistent with those previously reported by Gilbert et al (93 days) ${ }^{7}$ and by Ost (171 days). ${ }^{8}$ One explanation for the high mortality is that few, if any, of our patients had treatment for the underlying malignancy following IPC placement. The very short survival of our patients, as well as the patients in other studies, suggest that palliative care options should be considered in these patients and that IPCs should not be presented as a long term remedy for these patients. The main function of an IPC is the relief of dyspnea, improvement in the quality of life, reduced pleural procedures, decreased hospitalization rates and length of stay, rather than rates of spontaneous pleurodesis, which has been the focus of most prior studies. ${ }^{9}$ We argue that pleurodesis rates are inconsequential as an end point for future studies and agree with Ost et al that end points should be redefined to focus on more clinically meaningful outcomes. Pleurodesis rates do not matter if the median mortality is 90 days or less. In Ost's study, significant improvements in quality-adjusted life days (QALDs) and dyspnea were seen in patients with severe dyspnea at baseline and in patients on active treatment for their malignancy. Although dyspnea severity generally improved with IPC placement, QALDs and gain in utility were reported as only modestly improved. ${ }^{8}$ Given the poor outcome in the majority of these patients, it is reasonable to consider other alternatives for treatment when IPC placement is discussed. Also, these data suggest the use of IPCs for malignant pleural effusions may be most effective for symptom control when used in conjunction with therapy for the underlying malignancy.

Although it is easy to understand why the largest effusions ( $3 / 3$ volume) had a lower pleurodesis rate than the moderate size effusion ( $2 / 3$ volume), it is unclear why the moderate size effusions had a higher rate of pleurodesis than the smallest effusions (1/3 volume). This result might be a consequence of small sample size.
Our study has several limitations. The patient population is small, raising the possibility that conclusions are driven by outliers. Our results, however, do highlight trends in patient outcomes and are consistent with previously published studies. We were unable to directly confirm the date of death in some of our patients with benign effusions. This factor, combined with the small sample size, makes the mortality data in the benign effusion group less reliable than for the malignant effusion group. We were able only to confirm pleurodesis in the patients for which we had regular follow-up, and the actual rate of pleurodesis in our patients may be different from the information we obtained. Our interpretation of pleural volumes depended on the estimate of a single expert reader who was blinded as to outcome of the patients. The effect of effusion volumes on pleurodesis rates, while of interest, does not form the basis for our major conclusions.

Improvement in dyspnea occurred in up to $90 \%$ of patients with IPCs placed for malignant pleural effusion, thus leading to studies on the effectiveness of IPCs in the treatment of symptoms from benign pleural effusions. ${ }^{10}$ Indwelling pleural catheters have been evaluated as possible adjuncts to treatment in many conditions such as congestive heart failure, end stage liver disease, chylothorax, empyema, inflammatory pleurisy, renal disease and others. ${ }^{5,11}$ Herlihy et al reported their experience with IPC for the management of refractory pleural effusions in congestive heart failure and reported all of the patients improved from NYHA functional class IV to II with the use of IPCs. ${ }^{12}$ Our mortality data on patients with benign causes of pleural effusions suggest that recurrent pleural effusion is a harbinger for the end of life. The use of IPCs for benign conditions should be approached with caution given the poor prognosis in these patients as well. We suggest that future research should compare IPC with conventional palliative care and that IPCs should not be viewed as a definitive therapy for pleural effusions. Rather, IPC should be considered more as a bridge therapy to some other definitive treatment. In cases where some other definitive therapy does not exist, the cost of IPC therapy will have to be considered against the limited benefits. 
In addition to redefined end points, we think that an important feature of future research is appropriate follow-up. We suggest that these patients be seen at least on a monthly basis. If a patient does not make a follow-up appointment, then the patient and/or family should be contacted to determine how the patient is doing or whether the patient has died. Our study found that many of these patients did not live close to our hospital since these procedures were being done at a tertiary referral center. Given this demographic, there are problems maintaining an appropriate database, making it difficult to report reliable and accurate outcomes.

The mortality seen in our study is likely not due to the IPC itself, but rather due to the underlying diseases, especially since the survival in our patients with malignant pleural effusions is consistent with previously published data. As several earlier studies have concluded, there needs to be a consensus on how to define a successful endpoint in patients with an IPC placed for malignant or benign pleural effusions. Spontaneous pleurodesis is probably not the most important outcome to measure, but there is currently no objective measure for the assessment of dyspnea in these patients. It is also unreasonable to expect future studies to obtain meaningful subjective data without appropriate follow-up. Both appropriate follow up strategies and defined endpoints will make it easier to compare studies. Also, given the poor survival seen in these patients, conventional palliative care may need to be discussed prior to IPC placement since palliative care is very effective at controlling symptoms including dyspnea. Unless IPC placement can be shown to improve functional capacity or some other measure of the quality of life, it is unclear if the substantial costs of IPC placement are justified by the very short term benefits.

\section{REFERENCES}

1. Wrightson, John M., Davies, Helen E., Lee, Y.C. Gary. (2012). Pleural Effusion, Empyema, and Pneumothorax. In S.G. Spiro, G.A. Silvestri, A. Agustí. Clinical respiratory medicine. Philadelphia: Elsevier Saunders.

2. Cohen M, Sahn SA. Resolution of pleural effusions. Chest 2001;119:1547-1562.
Article citation: Copeland S, Schwalk A, Suchartlikitwong S, Yang S, Berdine G. Indwelling pleural catheters for recurrent pleural effusions: a useful clinical tool with serious implications. The Southwest Respiratory and Critical Care Chronicles 2018;6(25):8-13

From: The Department of Internal Medicine at the Texas Tech University Health Sciences Center in Lubbock, Texas

Submitted: $5 / 7 / 2018$

Accepted: $7 / 3 / 2018$

Reviewer: Victor Test MD

Conflicts of interest: none

This work is licensed under a Creative Commons Attribution-ShareAlike 4.0 International License

3. Chalhoub M, Harris K, Castellano M, et al. The use of the PleurX catheter in the management of non-malignant pleural effusions. Chron Respir Dis 2011;8:185-191.

4. Clive AO, Jones HE, Bhatnagar R, et al. Interventions for the management of malignant pleural effusions: a network meta-analysis. Cochrane Database Syst Rev 2016; CD010529.

5. Patil M, Dhillon SS, Attwood K, et al. Management of benign pleural effusions using indwelling pleural catheters: a systematic review and meta-analysis. Chest 2017;151:626-635.

6. Peikert T, Colby TV, Midthun DE, et al. Fibrosing mediastinitis: clinical presentation, therapeutic outcomes, and adaptive immune response. Medicine (Baltimore) 2011;90:412-423.

7. Gilbert CR, Lee HJ, Skalski JH, et al. The use of indwelling tunneled pleural catheters for recurrent pleural effusions in patients with hematologic malignancies: a multicenter study. Chest 2015;148:752-758.

8. Ost DE, Jimenez CA, Lei X, et al. Quality-adjusted survival following treatment of malignant pleural effusions with indwelling pleural catheters. Chest 2014;145:1347-1356.

9. Lee YC, Fysh ET. Indwelling pleural catheter: changing the paradigm of malignant effusion management. J Thorac Oncol 2011;6:655-657.

10. Bhatnagar R, Maskell NA. Indwelling pleural catheters. Respiration 2014;88:74-85.

11. Bhatnagar R, Corcoran JP, Fabien Maldonado F, et al. Advanced medical interventions in pleural disease. Eur Respir Rev 2016;25:199-213. Eur Respir Rev 2016;25:360.

12. Herlihy JP, Loyalka $P$, Gnananandh J, et al. PleurX catheter for the management of refractory pleural effusions in congestive heart failure. Tex Heart Inst J 2009;36:38-43. 